\title{
USING THE OPEN SOURCE LIBRARY LIBNODAVE FOR MONITORING TASKS IN THE SMART GRID SCENARIO
}

\author{
Isaías González Pérez, Antonio José Calderón Godoy, José María Portalo Calero, Manuel Calderón Godoy \\ Escuela de Ingenierías Industriales, Universidad de Extremadura, \{igonzp, ajcalde, jportalo, \\ calgodoy\}@unex.es
}

\begin{abstract}
Monitoring in the future intelligent power grids, called Smart Grids, is a critical task for the proper operation and surveillance of the infrastructure. On the other hand, open source systems are being progressively applied in $R \& D$ activities. This paper presents the utilization of the open source library Libnodave to communicate a programmable logic controller with a monitoring system through a TCP/IP network. The obtained results prove the feasibility of the proposal to be applied in the Smart Grids scenario.
\end{abstract}

Keywords: Smart Grid, Smart MicroGrid, monitoring, open source, Libnodave, TCP/IP.

\section{INTRODUCTION}

Smart Grids (SG) emerge as consequence of digitalization of traditional power grids and constitute the future enhanced networks based on distributed generation and distribution as well as penetration of renewable energy sources [12]. These infrastructures require advanced sensing, monitoring, control and communications. Indeed, SG are considered as a special type of Cyber-Physical System derived from the convergence of Information and Communication Technologies (ICT) and energy systems [6].

The capability of monitoring everything from power plants to consumer preferences is one of the main characteristics of $\mathrm{SG}$ [1]. In particular, proper monitoring implies an effective and reliable data exchange between control units and supervisory systems. The prevalent device for control and automation are Programmable Logic Controllers (PLC), which are configurable electronic devices hardened for industrial conditions.

In SG scenario, open source systems are an everincreasing trend as demonstrated by scientific publications. For instance, open source hardware devices like Arduino boards are applied to acquire signals from sensors in $[2,5,23]$. Concerning monitoring tasks, in [12] open source software, namely Easy Java/Javascript Simulations (EJS) is used to visualize real-time information of a Smart MicroGrid (SMG) operation. Other interesting examples can be found in [9].

This paper reports the usage of an open source library, Libnodave, to implement a TCP/IP interface in order to connect a PLC and a Visual Basic (VB)based monitoring system.

This work is a part of a $R \& D$ project about $S G$, namely regarding the deployment of a SMG and the creation of its digital replica. In this sense, the monitoring of physical equipment is a critical task for the correct operation, but also the main resource for data gathering. This data is the core of the digital replica, which is a simulation environment that accurately represents the dynamics of the real-world system [22].

This way, the developed interface is a stage to evaluate the feasibility of using open source systems to achieve proper data exchange and monitoring for the innovative scenario of SG/SMG.

The structure of the rest of the paper is as follows. Section 2 gives an overview of the involved elements. The third section deals with the developed interface, describing the functions provided by the open source library as well as the interconnection of the elements. The obtained results are reported in Section 4. Section 5 describes briefly an experimental SMG where the interface will be applied. Finally, the main conclusions are expounded to close the manuscript.

\section{BACKGROUND}

Visual Basic (VB) is an object-oriented programming language of Microsoft widely used to design Graphical User Interfaces (GUI) executed in PC. Indeed, it has also been applied to implement monitoring systems for renewable energy-based facilities [16, 20].

Libnodave is an open source library that enables the communication between PLC of manufacturer 
Siemens and various programming languages such as VB, C, C++, C\#, Delphi and Pascal. It can be used both for operative systems Microsoft Windows and Linux, which is an important feature given the wide heterogeneity of systems and equipment in automation networks [3, 26]. Recent publications dealing with cybersecurity issues have used such a library [17, 21], emphasizing its relevance in the automation and monitoring arena.

TCP/IP stands for Transmission Control Protocol/Internet Protocol, which is a family of protocols for communication over the network. TCP corresponds to the fourth level of the OSI model, e.g. transport layer, whereas IP is placed in the third level, the network layer. TCP/IP networks provide reliable data communication and are becoming the standard means for interconnection in both office and automation environments [4]. In these networks, information is shared between different nodes like computers, automation units, smart sensors and actuators, and so forth.

Concerning automation units, PLC are focused on industrial infrastructures which must operate reliable for decades [25], so they are highly configurable and hardened for industrial scenarios and, even, their lifetime is in the range of decades. Moreover, PLC are being introduced also in the challenging paradigm of Industry 4.0 [4, 7, 11, 13, 18, 19, 24]. In addition, their reliable and stable behaviour promote their application out of the scope of typical industrial facilities. In fact, recent examples of PLC-controlled infrastructures can be found in the domain of advanced industrial manufacturing [19, 24], renewable energy-based facilities $[10,14]$ and SG $[8$, 12].

\section{DEVELOPED INTERFACE}

The block diagram depicted in Figure 1 illustrates the developed system. On the one hand, a monitoring system is implemented using $\mathrm{VB}$, which runs in a PC. Its communication with a Siemens PLC is enabled by the library Libnodave. A TCP/IP network is used for data exchange between both nodes. The PLC corresponds to the S7-1200 series, a mid-range CPU with powerful capabilities, modular design and embedded Ethernet connectivity. In fact, a remarkable issue is that this series of PLC is not supported by the library according to the available information of its webpage [15].

The environment Microsoft Visual Studio has been chosen to manage the project of VB. The connected PLC corresponds to the model S7-1214. Concerning this device, TIA Portal V13 has been required to program the PLC code. It must be noted that the library Libnodave must be added to the Visual Studio project in order to use it.

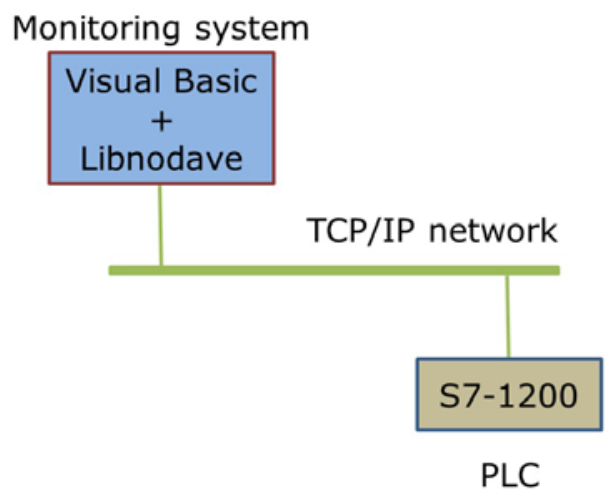

Figure 1: Block diagram of the implemented system

The Libnodave library includes the following functions:

- daveOSserialType, to establish the input/output channels in the operative system,

- openSocket, to create a TCP/IP connection with the device,

- daveInterface, to store the properties of a network of PLC,

- daveConnection, to store the characteristics of a particular PLC,

- daveReadBytes, to read bytes of data from a PLC memory,

- daveWriteBytes, to write bytes of data in memory or outputs of a PLC.

As a sample of the implemented code, some instructions are now given. To begin with, the $\mathrm{TCP} / \mathrm{IP}$ connection is established through this code:

Public Function Conectar(Optional ByVal Puerto As Integer $=102$

$\begin{array}{ll}\text { As String }=" 192.168 .0 .1 ", & \text { Optional ByVal IP } \\ \text { As Integer }=0, & \text { Optional ByVal Rack } \\ \text { As Integer }=1 \text { ) As Boolean } & \text { Optional ByVal Slot }\end{array}$

To implement write operations, e.g. to send data to the PLC memory from the user interface, the following instructions have been programmed:

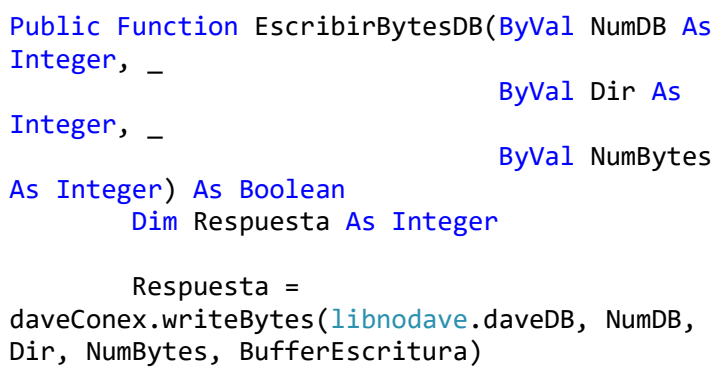




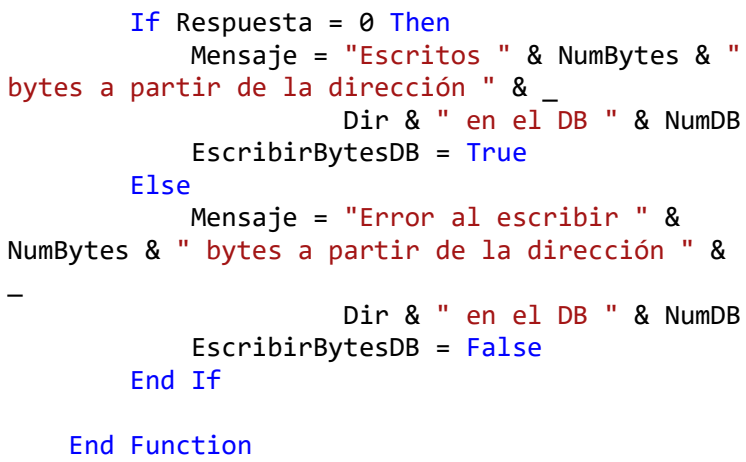

\section{RESULTS}

Once the configuration and programming of the involved nodes was accomplished, the obtained results allowed data exchange between the PLC and the monitoring system. The most illustrative screens of such monitoring system are provided in this section. It must be remarked that this system has been built as simple as possible in order to share information and test the suitability of the open source library and VB. Nevertheless, in further works the powerful functionalities of VB will be handled for an intuitive representation of the data.

Firstly, the overview of the main screen of the interface is shown in Figure 2. As it can be observed, it is composed by different sections, each one dedicated to perform certain operations that are commented from this point.

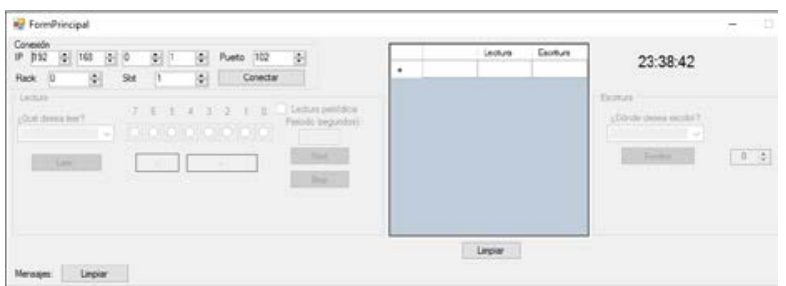

Figure 2: Main screen of the VB-interface

The TCP/IP connection parameters are configured through the following options: IP address, port, rack and slot. This way, the PLC is completely identified and the user is able to establish the connection between the nodes. The part of the interface devoted to this function is seen in Figure 3.

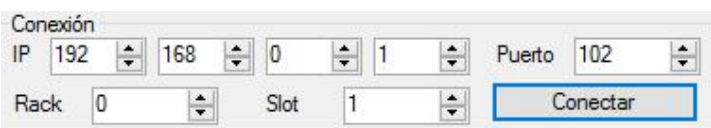

Figure 3: Configuration of TCP/IP parameters

Reading operations enable the visualization of data of the PLC, namely, the input signals and memory positions (data blocks and marks) can be observed in Figure 4. As can be seen, the user selects the

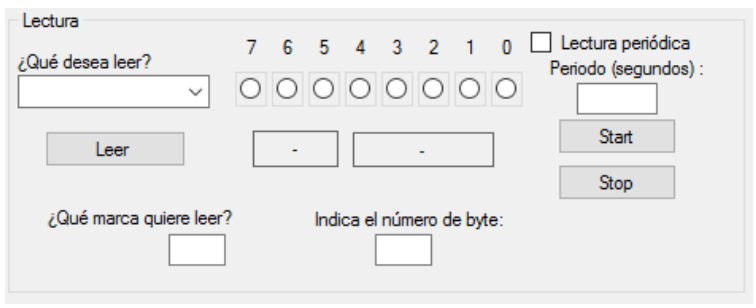

Figure 4: Section for read operations

To carry out write operations, the user can choose the data blocks and marks of the PLC memory as well as the rest of characteristics (Figure 5).

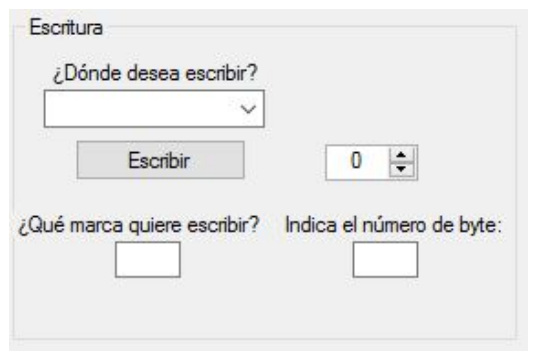

Figure 5: Section for write operations

Finally, a section of the interface is devoted to display in a table format the values that have been read and written from/in the PLC for a rapid inspection by the user (Figure 6).

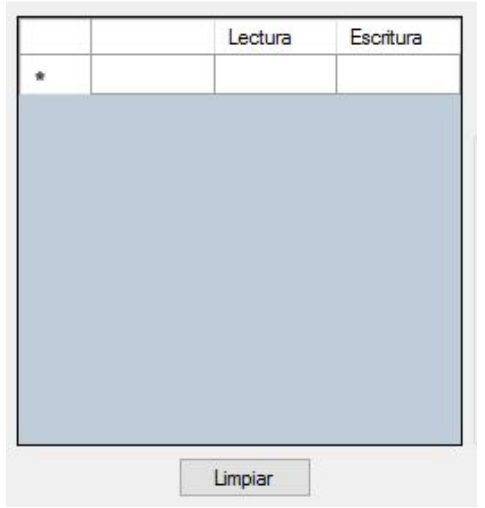

Figure 6: Table to illustrate read/write operations

On the view of the developed system, an effective data exchange between the PLC and the VB-based monitoring system can be established via a TCP/IP network thanks to the open source library Libnodave. 


\section{SMG DESCRIPTION}

As it was mentioned in the Introduction, the developed interface is envisioned to be applied for SG/SMG. In particular, a SMG within a R\&D project is the environment of further application. This SMG is briefly described in this section.

The SMG integrates electrical generation through monocrystalline photovoltaic (PV) modules and a hydrogen fuel cell (FC) with consumption by means of a hydrogen electrolyzer (HE) and loads. A lithiumion battery acts as short-term energy storage to host the electrical flows between the aforementioned components. On the other hand, a set of hydrogen bottles store the hydrogen produced by the HE and feed it to the FC. The main goal of this micro-grid is to supply the loads using the photovoltaic modules and to generate hydrogen if there is available photovoltaic energy. In the opposite situation, if there is no solar irradiance, the accumulated hydrogen is used by the FC to fulfil the demand of the connected loads. To handle these energy flows and interactions, a set of sensors and actuators (S/A) as well as an automation system have been deployed. In addition, a monitoring system is required to supervise the proper operation of the SMG. Figure 7 depicts the block diagram of the SMG.

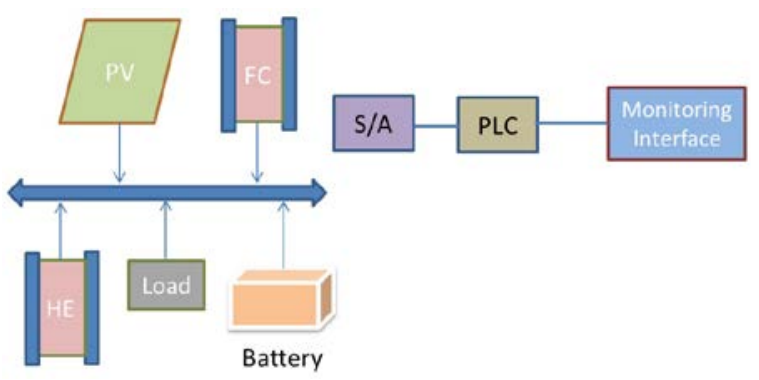

Figure 7: Block diagram of the SMG where the interface will be applied

\section{CONCLUSIONS}

SG are innovative networks of distributed power sources and consumers that impose the collection, processing and visualization of large amount of data. Hence, monitoring systems are a vital part of SG. Given this scenario, this work has presented the development of a monitoring system using VB and the open source Libnodave. The latter one is a free software tool for different programming languages that provides deep configuration capability and, facilitates the deployment of monitoring systems/interfaces.

In fact, the proposed system is framed in a $R \& D$ project about the implementation of a SMG based on renewable energy and its digital replication, which relies in massive data retrieving, being the monitoring system a crucial element.

Particularly, information from a Siemens PLC has been successfully shared with the monitoring system through a TCP/IP communication network.

Future works include the exchange of data with a network of PLC and also the visualization of analogue signals through graphical charts. Cybersecurity issues will also be studied given the networked environment and the increasing threats.

\section{Acknowledgments}

This research has been funded by the project IB18041 supported by the Junta de Extremadura in the VI Plan Regional de I+D+i (2017-2020), cofinanced by the European Regional Development Funds FEDER (Programa Operativo FEDER de Extremadura 2014-2020).

\section{References}

[1] Alvial-Palavicino, C., Garrido-Echeverría, N., Jiménez-Estévez, G., Reyes, L., Palma-Behnke, R., (2011) "A methodology for community engagement in the introduction of renewable based smart microgrid", Energy for Sustainable Development, vol. 15, pp. 314-323.

[2] Batista, N.C., Melício, R., Mendes, V.M.F., (2014) "Layered Smart Grid architecture approach and field tests by ZigBee technology", Energy Conversion and Management, vol. 88, pp. $49-59$.

[3] Botta, A., de Donato, W., Persico, V., Pescapé, A., (2016) "Integration of Cloud computing and Internet of Things: A survey", Future Generation Computer Systems, vol. 56, pp. 684700. DOI: 10.1016/j.future.2015.09.021.

[4] Calderón, A.J., González, I., (2018) “Integration of Sensor and Actuator Networks and the SCADA System to Promote the Migration of the Legacy Flexible Manufacturing System towards the Industry 4.0 Concept", Journal of Sensor and Actuator Networks, vol. 7(2), 23.

[5] Calderón, A.J., González, I., Calderón, M., Segura, F., Andújar, J.M., (2016) "A New, Scalable and Low Cost Multi-Channel Monitoring System for Polymer Electrolyte Fuel Cells", Sensors, vol. 16(3), 349. 
[6] Camarinha-Matos, L.M., (2016) "Collaborative smart grids - A survey on trends", Renewable and Sustainable Energy Reviews, vol. 65, pp. 283-294, DOI: 10.1016/j.rser.2016.06.093

[7] Chen, J., Tai, K.C., Chen, G.C., (2017) "Application of Programmable Logic Controller to Build-up an Intelligent Industry 4.0 Platform", Procedia CIRP, vol. 63, pp. 150155.

[8] Cintuglu, M.H., Mohammed, O.A., Akkaya, K., Uluagac, A.S., (2016) "A Survey on Smart Grid Cyber-Physical System Testbeds", IEEE Communication Surveys and Tutorials, vol. 19, pp. 446-464.

DOI: 10.1109/COMST.2016.2627399.

[9] Costa, D.G., Duran-Faundez, C., (2018) “OpenSource Electronics Platforms as Enabling Technologies for Smart Cities: Recent Developments and Perspectives", Electronics, vol. 7, pp. 404.

[10] Dispenza, G., Sergi, F., Napoli, G., Randazzo, N., Di Novo, S., Micari, S., Antonucci, V., Andaloro, L., (2017) "Development of a solar powered hydrogen fueling station in smart cities applications", International Journal of Hydrogen Energy, vol. 42(46), pp. 2788427893. DOI: 10.1016/j.ijhydene.2017.07.047.

[11] González, I., Calderón, A.J., (2018) "Development of Final Projects in Engineering Degrees around an Industry 4.0-Oriented Flexible Manufacturing System: Preliminary Outcomes and Some Initial Considerations", Education Sciences, vol. 8, 214.

[12] González, I., Calderón, A.J., Andújar, J.M., (2017) "Novel Remote Monitoring Platform for RES-Hydrogen based Smart Microgrid", Energy Conversion and Management, vol. 148, pp. 489-505.

DOI: 10.1016/j.enconman.2017.06.031.

[13] González, I., Calderón, A.J., Figueiredo, J., Sousa, J.M.C., (2019) "A Literature Survey on Open Platform Communications (OPC) Applied to Advanced Industrial Environments", Electronics, vol. 8, pp. 510.

[14] González, I., Ramiro, A., Calderón, M., Calderón, A.J., González, J.F., (2012) "Estimation of the state-of-charge of gel leadacid batteries and application to the control of a stand-alone wind-solar test-bed with hydrogen support", International Journal of Hydrogen
Energy, vol. 37(15), pp. 11090 - 11103. DOI: 10.1016/j.ijhydene.2012.05.001.

[15] http://libnodave.sourceforge.net/

[16] Huda, A.S.N., Zivanovic, R., (2017) "Largescale integration of distributed generation into distribution networks: Study objectives, review of models and computational tools", Renewable and Sustainable Energy Reviews, vol. 76, pp. 974-988.

[17] Kleinmann, A., Wool, A., (2014) "Accurate Modeling of the Siemens S7 SCADA Protocol for Intrusion Detection and Digital Forensics", Journal of Digital Forensics, Security and Law, vol. 9 , pp. 37-50.

[18] Langmann, R., Rojas-Peña, L.F. A PLC as an Industry 4.0 component. Proceedings of the 13th International Conference on Remote Engineering and Virtual Instrumentation (REV), Madrid, Spain, 24-26 February 2016.

[19] Legat, C., Vogel-Heuser, B., (2017) "A configurable partial-order planning approach for field level operation strategies of PLC-based industry 4.0 automated manufacturing systems", Engineering Applications of Artificial Intelligence, vol. 66, pp. 128-144.

[20] Madeti, S.R., Sing, S.N., (2017) "Monitoring system for photovoltaic plants: A review", Renewable and Sustainable Energy Reviews, vol. 67 , pp. 1180-1207.

[21] Martin-Liras, L., Prada, M.A., Fuertes, J.J., Morán, A., Alonso, S., Domínguez, M., (2017) "Comparative analysis of the security of configuration protocols for industrial control devices", International Journal of Critical Infrastructure Protection, vol. 19, pp. 4-15.

[22] O’Dwyer, E., Pan, I., Acha, S., Shah, N., (2019) "Smart energy systems for sustainable smart cities: Current developments, trends and future directions", Applied Energy, vol. 237, pp. 581597.

[23] Oprea, S.-V., Bâra, A., Uță, A.I., Pîrjan, A., Căruțașu, G., (2018) “Analyses of Distributed Generation and Storage Effect on the Electricity Consumption Curve in the Smart Grid Context", Sustainability, vol. 10, pp. 2264.

[24] Pisching, M.A., Pessoa, M.A.O., Junqueira, F., Filho, D.J., Miyagi, P.E., (2018) "An architecture based on RAMI 4.0 to discover equipment to process operations required by 
products", Computers and Industrial Engineering, vol. 125, pp. 574-591.

[25] Simons, S., Abé, P., Neser, S., (2017) "Learning in the AutFab - the fully automated Industrie 4.0 learning factory of the University of Applied Sciences Darmstadt", Procedia Manufacturing, vol. 9, pp. 81-88.

[26] Wollschlaeger, M., Sauter, T., Jasperneite, J., (2017) "The Future of Industrial Communication. Automation Networks in the Era of the Internet of Things and Industry 4.0", IEEE Industrial Electronics Magazine, vol. 11(1), pp. 17-27. DOI: 10.1109/MIE.2017.2649104.

(C) (3) 2019 by the authors. BY NC SA open access publication under the terms and conditions of the Creative Commons Attribution CC BY-NC-SA 4.0 license (https://creativecommons.org/licenses/bync-sa/4.0/deed.es). 